\title{
Análise de desempenho de redes ópticas híbridas WDM/OCDM
}

\section{Performance analyses of hybrid WDM/OCDM optical networks}

\author{
Fábio Renan Durand ${ }^{1}$; Edson Moschim²
}

Resumo

\begin{abstract}
Neste trabalho apresenta-se uma análise de redes ópticas híbridas multiplexadas em comprimentos de onda e códigos ópticos WDM/OCDM (Wavelength Division Multiplexing/Optical Code-Division Multiplexing). Nesta análise foram apresentadas as vantagens do emprego de caminhos ópticos virtuais compostos por códigos ópticos ortogonais OOC (Orthogonal Optical Codes) e comprimentos de onda para a comutação e roteamento no domínio óptico, bem como a arquitetura de rede utilizada. Como resultados obtidos observou-se que o emprego de códigos OOC juntamente com conversores de códigos ópticos tem um grande impacto na diminuição da probabilidade de bloqueio de estabelecimento de caminhos ópticos virtuais, assim aumenta-se a utilização da rede.

Palavras-chave: Redes ópticas. WDM/OCDM. Wavelength Division Multiplexing/Optical Code-Division Multiplexing. OOC. Orthogonal Optical Codes.
\end{abstract}

\begin{abstract}
In this work, optical networks using the hybrid technology of wavelength division multiplexing and optical code division multiplexing (WDM/OCDM) are investigated. The architecture of the network is described and it is shown the main advantages of applied optical paths with wavelengths and optical orthogonal codes (OOC) to routing and switching virtual optical paths. The main results show that the use of OOC codes together with optical codes converters have a great impact on the decrease of blocking probability for establishing virtual optical paths, increasing network use.

Key words: Optical Networks. WDM/OCDM. Wavelength Division Multiplexing/Optical Code-Division Multiplexing). OOC. Orthogonal Optical Codes.
\end{abstract}

\footnotetext{
1 Professor no Dep. de Engenharia Elétrica da Universidade Estadual de Londrina-UEL e Universidade Norte do Paraná- UNOPAR Titulação: Mestre em Engenharia Elétrica (USP/São Carlos) e Doutorando em Engenharia Elétrica. Universidade Estadual de Campinas. E-mail: frdurand@uel.br.

2 Professor Doutor da Faculdade de Engenharia Elétrica e Computação - FEEC - Universidade Estadual de Campinas - UNICAMP. Campinas - SP.
} 


\section{Introdução}

O crescimento rápido e global de tráfego em função da explosão da popularidade da Internet está acelerando o desenvolvimento e a implementação da tecnologia de multiplexação por divisão de comprimentos de onda WDM (Wavelength Division Multiplexing) como uma forma de explorar a elevada capacidade de transmissão das fibras ópticas (MURTHY; GURUSAMY, 2002). Esta tecnologia evoluiu de meio físico de conexão ponto-a-ponto e passou a ser utilizada como camada de transporte (LIU et. al., 2002). As redes ópticas que empregam a tecnologia da camada de transporte WDM estabelecem caminhos ópticos (ligthpaths) entre os nós por meio de crossconects ópticos e, dessa forma, obtém-se transparência, elevada capacidade de transmissão e escalabilidade (MURTHY; GURUSAMY, 2002). Um aspecto importante deste tipo de rede é o gerenciamento do estabelecimento dos ligthpaths, pois os comprimentos de onda consistem de um elemento limitado em função de fatores como: a estabilidade de fontes ópticas, o espaçamento limitado na região de amplificação dos amplificadores EDFA (Erbium Doped Fiber Amplifier) e espaçamento limitado entre os canais ópticos devido ao crosstalk induzindo por filtros ópticos não ideais (DURAND et. al., 2004). Esses fatores atuam de forma distinta nas configurações de redes ópticas existentes. Nas redes WAN (Wide Area Network), observa-se que a limitação do número de comprimentos de onda ocorre em função da estabilidade das fontes ópticas que limitam o espaçamento entre os canais na região de amplificação dos amplificadores EDFA (BANERJEE et. al., 2001). Por outro lado, nas redes MANs (Metropolitan Area Network) e, principalmente em LANs (Local Area Network), a principal limitação é o fato do número de comprimentos de onda ser limitado a aproximadamente ao número de nós da rede (KITAYAMA; WADA; SOTOBAYASHI, 2000). Nas redes LAN e MAN, também se observam a limitação em função da estabilidade das fontes ópticas e crosstalk, pois nestas redes procura- se utilizar-se dispositivos de baixo custo com características técnicas que limitam o seu desempenho. Uma alternativa no caso de redes LAN e MAN é o emprego da tecnologia de acesso múltiplo por divisão de código OCDMA (Optical CodeDivision Multiple Access). Na tecnologia OCDMA cada bit é dividido em $n$ intervalos de tempo denominados chips e uma sequiência de chips forma uma assinatura denominada codeword, onde cada usuário da rede OCDMA possui uma assinatura distinta. Dessa forma, todos os bits "1" a serem transmitidos são codificados no formato da assinatura e os bits "0" não são transmitidos ou transmite-se o complemento da assinatura (MAJUMDER; AZHARI; ABBOU, 2005). O sinal codificado é transmitido para todos os nós da rede e o crosstalk entre os usuários ocorre principalmente pela interferência de acesso múltiplo MAI (MultipleAccess Interference) (ZHANG; JI; CHEN, 1999). O OCDMA pode operar de forma assíncrona, sem controle centralizado, sem colisões, com baixo tempo de atraso e existe a possibilidade de oferecer QoS (Quality of Service) na camada física. Porém, mesmo tendo em vista o constante desenvolvimento de redes OCDMA, a tecnologia de multiplexação por divisão de código OCDM (Optical CodeDivision Multiplexing) empregada como rede de transporte não está sendo largamente explorada (KITAYAMA; WADA; SOTOBAYASHI, 2000) e (CINCOTTI, 2004). Uma alternativa é a utilização de redes híbridas WDM/OCDM. Nessas redes, a camada de transporte é composta por caminhos ópticos virtuais baseados em comprimento de onda e códigos ópticos. Esse tipo de rede vem apresentando constante evolução em função de sua utilização em conjunto com a tecnologia GMPLS (Generalized Multiprotocol Label Switching) que realiza a integração entre os planos de controle da rede óptica e protocolo de Internet (IP) (KITAYAMA; WADA, 2003), por outro lado está ocorrendo um desenvolvimento de técnicas de construção de códigos OOC que aumentam o seu desempenho em aplicações como as redes estudadas neste trabalho (MAJUMDER; AZHARI; ABBOU, 2005).

Neste contexto, realiza-se neste trabalho uma 
análise de redes ópticas híbridas que empregam as tecnologias WDM e OCDM, onde o principal objetivo é ilustrar como o emprego destas tecnologias pode aumentar a capacidade e a versatilidade das redes ópticas. Também será investigado o impacto do uso de conversores de códigos ópticos nestas redes. $\mathrm{O}$ restante deste artigo está organizado da seguinte forma: a seção Arquitetura e Funcionamento da Rede ilustra a evolução e a arquitetura das redes ópticas híbridas WDM/OCDM; na seção Materiais e Métodos, apresenta-se a metodologia utilizada na análise do desempenho das redes; na seção Resultados e Discussão são ilustrados os principais resultados; finalmente, na seção Conclusões são discutidas as principais conclusões obtidas no trabalho.

\section{Arquitetura e Funcionamento da Rede}

\section{Evolução Histórica}

As primeiras aplicações de redes ópticas WDM/ OCDM foram inicialmente propostas e analisadas em Kitayama, Wada e Sotobayashi (2000). Neste trabalho, investigou-se a utilização de caminhos ópticos virtuais baseados em códigos ópticos bipolares, nestes códigos realiza-se a codificação por alteração de fase. No estabelecimento destes caminhos ópticos, realizava-se a conversão de códigos ópticos, porém a restrição da conversão de comprimento de onda era mantida. O foco desta análise estava relacionado com o desempenho dos conversores de códigos ópticos bipolares adicionados aos crossconnects. Assim, as principais investigações foram com relação ao número de códigos ópticos disponíveis na rede e a interferência causada entre códigos ópticos de diferentes comprimentos de onda. Em Zhang, Ji e Chen (1999) foi proposta a utilização de roteamento de redes ópticas de pacotes WDM baseado em códigos ópticos ortogonais OOC (Optical Orthogonal Code). Porém, somente considerações sobre a possibilidade da implementação foram discutidas juntamente com possíveis soluções teóricas. Já em Kitayama, Wada e Sotobayashi
(2000), foi proposta a utilização de códigos ópticos bipolares como labels ópticos para o roteamento de protocolo IP mapeado diretamente sobre a camada óptica. Nesta configuração, utilizava-se a correlação óptica dos códigos para efetuar o roteamento por meio de comutadores ópticos de labels PLSR (Photonic Label Switching Router). Estes roteadores eram compostos por processadores e conversores de labels ópticos. O emprego de roteamento por labels passou a ser apontado como uma forma de aumentar a capacidade de comutação dos roteadores, pois nesta tecnologia a informação transmitida se mantém totalmente no domínio óptico e só o label é processado eletronicamente ou opticamente (BLUMENTHAL et al., 2000) e (MURTHY; GURUSAMY, 2002). Outra vantagem da utilização de labels é a possibilidade de integração entre o plano de controle IP e a camada óptica WDM por meio do GMPLS (BANERJEE et al., 2001). Seguindo a tendência do emprego de códigos ópticos como labels, foi proposto em (WEN et al., 2002) uma arquitetura OMPLS (Optical - MPLS) onde os labels eram compostos de códigos ópticos OOC e comprimentos de onda. Nesta arquitetura, todo o processamento dos labels é realizado no domínio óptico. Em (KITAYAMA; WADA, 2003) foi proposta e analisada uma arquitetura de roteamento baseada em labels compostos por códigos ópticos bipolares. Nesta arquitetura, o sistema de comutação pode ser na forma de pacotes, rajadas ou circuitos. A principal vantagem desta arquitetura é que o processamento dos labels é realizado no domínio óptico e sua velocidade de processamento é limitada somente pelo tempo de propagação no correlator óptico. Portanto, analisando os trabalhos que englobam redes WDM/OCDM, observa-se que ainda não foi focado o desempenho global destas redes em função de parâmetros como o número códigos ópticos, comprimentos de onda e o número de conversores de códigos ópticos que é o objetivo deste trabalho. 


\section{Principio da Multiplexação Óptica por Divisão de Código (OCDM)}

A tecnologia OCDM possui analogia com a tecnologia de spread spectrum de múltiplo acesso por divisão de código, CDMA (Code Division Multiplexing Access), empregada em comunicações sem fio. Porém, em contraste com as técnicas baseadas no espalhamento em freqüência adotadas em CDMA, na tecnologia OCDM emprega-se o espalhamento no tempo (KITAYAMA; WADA; SOTOBAYASHI, 2000). Em geral, os esquemas de codificação OCDM são classificados em coerentes e não coerentes, dependendo do grau de coerência da fonte de luz empregada. Porém, a principal diferença entre estes sistemas é que nos sistemas não coerentes emprega-se códigos ópticos unipolares que são obtidos por modulação em intensidade, já nos sistemas coerentes emprega-se códigos ópticos bipolares que são obtidos por modulação por mudança de fase. Assim, os códigos unipolares apresentam uma pseudo-ortogonalidade e possuem uma relação sinal/ruído de interferência menor, SIR (signal-tointerference noise ratio) que os códigos bipolares empregados em sistemas coerentes. Esta característica fornece um ganho muito alto de processamento aos sistemas coerentes, porém as dificuldades de implementação dos sistemas coerentes os tornam ainda inviáveis. A seleção do tipo de código empregado é um dos elementos-chave do OCDM, pois os códigos devem apresentar baixa correlação cruzada para aumentar a isolação entre os canais. Diversas formas de códigos vêm sendo estudadas e empregadas, como códigos ópticos ortogonais OOC (Optical Orthogonal Codes), códigos primos, sequiência M, entre outros (ZHANG; JI; CHEN, 1999). Os códigos OOC são largamente empregados em função de seus valores de autocorrelação e correlação cruzada (ZHANG; JI; CHEN, 1999) (KITAYAMA; WADA; SOTOBAYASHI, 2000) e (DJORDJEVIC et al., 2004). Neste tipo de codificação, os bits transmitidos são modulados por OOC no domínio do tempo, assim para cada bit "1" que é transmitido um código OOC é enviado e quando o bit " 0 " é transmitido nenhum código é enviado ou envia-se a forma complementar do código OOC (MAJUMDER; AZHARI; ABBOU, 2005). Neste trabalho, os códigos OOC são empregados para formar em conjunto com os comprimentos de onda os caminhos ópticos virtuais que serão roteados e encaminhados na rede. $\mathrm{O}$ crosstalk entre os códigos OOC, neste caso, é fornecido principalmente pela interferência de acesso múltiplo MAI (Multiple-Access Interference), porém em algumas configurações de roteadores (WEN et al., 2002) empregam-se dispositivos para efetuar a sua supressão. Neste trabalho, serão empregados códigos com peso 5 e 10, e comprimento 800 e 1500, respectivamente. O peso do código representa a quantidade de chips "1" transmitidos e o comprimento é dado pelo numero de chips " 1 "e “0”. Para esta configuração de códigos, já se demonstrou roteadores que suportam 12 códigos por comprimento de onda com uma taxa de erro de conversão de código aceitável de $10^{-9}$ (WEN et al., 2002).

\section{Estrutura do Roteador}

A estrutura básica do roteador óptico é ilustrada na Fig. 1 (a). Este tipo de roteador caracteriza-se por processar os comprimentos de onda e códigos OOC no domínio óptico, desta forma a leitura e troca de labels são feitas de forma ultra-rápida (ZHANG; JI; CHEN, 1999) . Na Fig. 1(b), é detalhado como ocorre o processo de leitura e troca dos labels. A informação que chega ao roteador vem rotulada pelo comprimento de onda e pelo código OOC. O código OOC será decodificado pelo filtro óptico que fará a correlação e o encaminhamento para a interface de saída onde deve ser comutados a informação e o label. Esta estrutura genérica é melhor detalhada em (KITAYAMA; WADA; SOTOBAYASHI, 2000) e (KITAYAMA; WADA, 2003). 


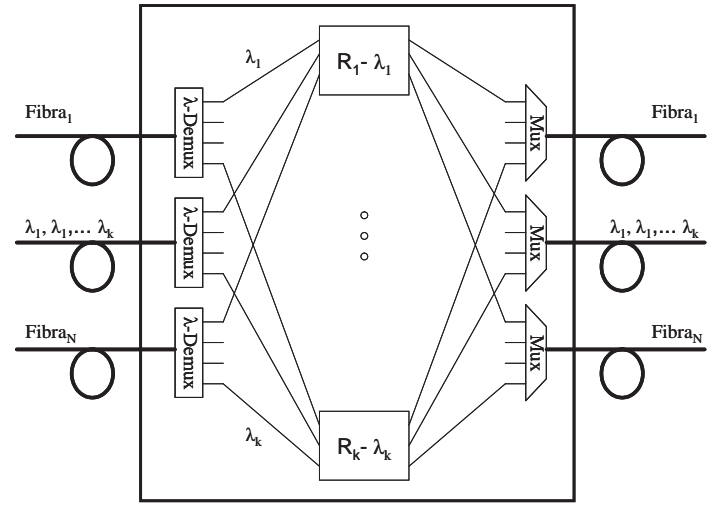

(a)

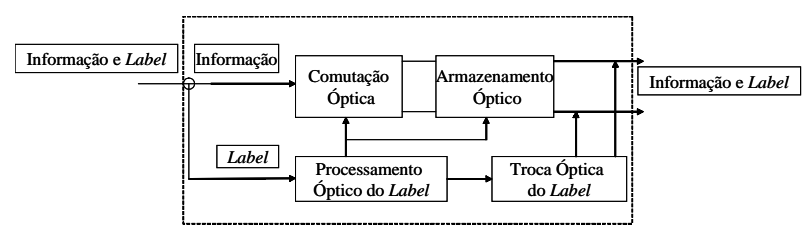

(b)

Figura 1. (a) Roteador Óptico (b) Núcleo do Roteador $\left(R_{i}\right)$.

Atualmente, o conversor de códigos ópticos tem sido utilizado nas redes híbridas WDM/OCDM, com a finalidade de aumentar o desempenho das mesmas. Este dispositivo consiste de uma configuração de SOAs (Semiconductor Optical Amplifier), na qual o código OOC de entrada pode ser alterado de forma óptica, pelo fornecimento de outro código OOC na sua saída (DURAND et al., 2005). O seu uso faz com que se elimine a restrição de manter a continuidade do mesmo código óptico ao longo de todo o caminho virtual estabelecido.

\section{Materiais e Métodos}

Nesta etapa de trabalho, a análise de desempenho das redes ópticas emprega o modelo analítico proposto em Barry e Humblet (1996) para AON (All Optical Network). Esse modelo foi aqui estendido para o caso de redes que empregam o roteamento por caminhos ópticos virtuais formados por códigos OOC e comprimento de onda (DURAND et al., 2004), e sua escolha resulta da simplicidade e precisão nos resultados, quando comparado com outros métodos analíticos.

O modelo geral proposto por (BARRY; HUMBLET, 1996) considera que uma requisição de tráfego de um nó A para um nó B, como ilustra a Fig. 2, é baseado nos de parâmetros $P_{l}$ e $P_{n}$ que são definidos como:

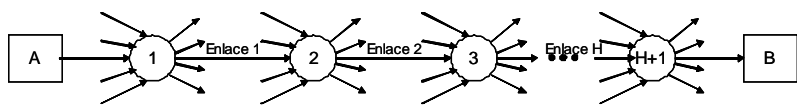

Figura 2. Ilustração de uma conexão entre o nó A e o nó B.

$P_{l}-$ Probabilidade que a requisição deixe a trajetória em um nó intermediário.

$P_{n}$ - Probabilidade que uma nova requisição seja realizada em um nó num comprimento de onda disponível.

As considerações seguintes devem ser observadas para o desenvolvimento do modelo de obtenção da probabilidade de bloqueio de estabelecimento de caminhos ópticos virtuais:

- o comprimento de onda $(\lambda)$ é dito disponível, se não estiver sendo utilizado nos enlaces $i$ ou enlace $i$ -1 , ou se estiver sendo liberado neste enlace;

- todos os eventos que ocorrerem em diferentes comprimentos de onda são independentes;

- a probabilidade que $\lambda$ seja utilizado no enlace 0 é $\rho_{0} \stackrel{\text { def }}{=} 0$;

- dado o estado do enlace $i-\lambda$, isto é, se l é utilizado ou não, o estado do enlace é estatisticamente independente dos estados dos outros enlaces;

- dado que 1 é utilizado no enlace $i-\lambda$, assume-se que a seção sobre $\lambda$ deixa a percurso no nó $i$ com probabilidade $P_{l}$. Portanto, continua no mesmo comprimento de onda. Todas as seções deixam o percurso no nó $H+\lambda$;

- se 1 não é utilizado no enlace $i-\lambda$, então uma nova seção junta-se no nó $i$ sobre 1 com probabilidade $P_{n}$. Nenhuma sessão junta-se ao nó $H+\lambda$;

- se 1 não é utilizada no enlace $i-\lambda$ e a sessão que está utilizando 1 neste enlace deixa no nó $i$, então 
uma nova seção junta-se ao nó $i$ sobre $\lambda$ com probabilidade $P_{n}$. Nenhuma requisição é aceita no nó $H+1$.

Em função das considerações feitas, a probabilidade de bloqueio é dada por :

$P_{B}\left(\lambda\right.$ utilizado em $i^{1} / 2 \lambda$ não utilizado em $\left.i-1\right)=P_{n}$ $P_{B}\left(\lambda\right.$ utilizado em $i^{1 / 2} \lambda$ utilizado em $\left.i-1\right)=\left(1-P_{l}\right)$ $+P_{l} P_{n}$

em que a primeira equação é a definição de $P_{n}$ e a segunda equação acompanha o fato que se $\lambda$ é utilizado no enlace $i-1$, então ele é utilizado no enlace $i$ se a requisição continua na trajetória ou se deixar a trajetória e novas requisições forem realizadas. Observe que $P_{l}=1$ representa que o comprimento de onda é utilizado em sucessivos links, independentemente.

Considerando os elementos discutidos acima, será particularizada a probabilidade de bloqueio de uma rede comutada por caminhos ópticos virtuais com diferentes graus de conversões de códigos ópticos OOC. Nesta rede consideram-se $F$ fibras com $W$ comprimentos de onda em cada fibra, $H$ é o número de enlaces e $M$ códigos ópticos por comprimento de onda. Por simplicidade será considerado tráfego uniforme em cada caminho óptico virtual.

Caminho óptico virtual sem conversão de comprimento de onda e código óptico: inicialmente será considerado o caso em que não há conversão óptica ao longo da rota do caminho óptico virtual. Assim, o estabelecimento das conexões entre os nós deve considerar a restrição de continuidade de código e comprimento de onda. Este esquema é similar ao roteamento fixo, ou seja, a seção será bloqueada se todos os caminhos ópticos virtuais LSPs (Label Switched Path) forem bloqueados. Assumindo que cada caminho óptico virtual possua uma carga $\rho$, a probabilidade de bloqueio média da rede é dada por:

$P_{B}=\left(1-\left[1-\left(1-\frac{1}{F}\right) P_{n}\right]^{H}\right)^{W x M}$ em que $P_{n}=\frac{\rho}{L-\rho(L-1)}$,

$L$ representa o número de enlaces compartilhados pela mesma seção simultaneamente e é denominado comprimento de interferência. Este parâmetro é função da topologia e do algoritmo de roteamento. Neste trabalho será considerado um $L$ médio em função do número de enlaces compartilhados em função da topologia.

Caminho óptico virtual sem conversão de comprimento de onda: por outro lado, se houver a conversão de código óptico continuará havendo a restrição de continuidade do mesmo comprimento de onda em todas as conexões do caminho óptico virtual. Assim, para redes que empregam conversores de códigos, considera-se que todos os códigos ópticos que estão no mesmo comprimento de onda no mesmo enlace como sendo um trunk. Desta forma, o trunk é dito ocupado se todos os seus códigos ópticos estão ocupados e uma sequência de trunks ao longo da trajetória entre nó de origem e nó de destino forma um caminho óptico virtual com probabilidade de bloqueio dada por:

$P_{B}=P\{$ todos comprimentos de onda sem conversão estão bloqueados $\}$

$P_{B}=\prod_{f n=1}^{F} P\{$ Caminho virtual trunk $f$ bloqueado $\}$ (2)

Nesta formulação, pode-se verificar que a probabilidade de bloqueio de cada trajetória-tronco $n$ é igual a probabilidade de bloqueio como no caso de roteamento de comprimentos de onda derivado em (SUBRAMANIAM; AZIZOGLU; SOMANI, 1996). Portanto, a probabilidade de bloqueio considerada quando somente a conversão de códigos ópticos é permitida é dada por:

$P_{B}=\left(\left(1-\left[1-\left(1-\frac{1}{F}\right) P_{n}\right]^{M}\right)^{H}\right)^{W}$ 
Por meio desta formulação, fica claro que o problema de roteamento em duas dimensões (comprimento de onda/código) pode ser solucionado de forma análoga a uma dimensão como o roteamento de comprimento de onda. Porém, não é viável fornecer a capacidade de conversão de códigos ópticos a todos os nós da rede, como foi ilustrado na literatura para o caso de conversores de comprimento de onda (SUBRAMANIAM; AZIZOGLU; SOMANI, 1996). Dessa forma, os conversores de códigos ópticos são distribuídos de forma esparsa pela rede. Assim, será considerado que nós com capacidade de conversão de códigos ópticos estão uniformemente distribuídos pela rede. Neste caso, haverá $N$ troncos independentes e com restrição de conversão de comprimento de onda e pode-se considerar que as rotas entre os nós de origem e destino serão divididas em $K$ sub-rotas com probabilidade de bloqueio independentes. Considerouse que os conversores estão uniformemente distribuídos pela rede por ser a melhor configuração para tráfego uniforme (SUBRAMANIAM; AZIZOGLU; SOMANI, 1996) e (DURAND et al., 2005). A probabilidade de bloqueio é dada, neste caso, por:

$$
P\left\{S^{k}=1\right\}=\left(\left(1-\left[1-\left(1-\frac{1}{F}\right) P_{n}\right]^{q}\right)^{M}\right)^{K}
$$

Para as requisições subsequentes, o seu bloqueio ocorrerá se todos os $N$ troncos estiverem bloqueados. Dessa forma, a probabilidade de bloqueio é dada por:

$$
P_{B}=\prod_{n=1}^{N}\left(1-P\left\{S^{k}=1\right\}\right)=\left(1-\left(\left(1-\left[1-\left(1-\frac{1}{F}\right) P_{n}\right]^{q}\right)^{W}\right)^{K}\right)^{N}
$$

onde $K$ é o número de sub-rotas, $q=H / K$ é o número de enlaces na sub-rota, e $P\left\{S^{k}=1\right\}$ representa a probabilidade de bloqueio da sub-rota $k$. Esta equação apresenta resultados precisos quando $H$ é múltiplo de $K$, caso contrário representa uma aproximação da probabilidade de bloqueio (MURTHY; GURUSAMY 2002) e (DURAND et al., 2005). Por meio dessa formulação, evidencia-se o problema de roteamento em duas dimensões (comprimento de onda/código) pode ser solucionado de forma análoga a uma dimensão como o roteamento de comprimento de onda.

\section{Resultados e Discussão}

A análise de resultados tem início com o cálculo da probabilidade de bloqueio do estabelecimento de caminhos ópticos virtuais, considerando a restrição da continuidade de comprimento de onda e códigos ópticos. A Fig. 3 ilustra a probabilidade de bloqueio em função da carga do link para uma rede com 8 enlaces e 8 comprimentos de onda. Em todos os resultados apresentados, considera-se somente uma fibra óptica. Como, neste trabalho, os comprimentos de onda e os códigos OOC estabelecem caminhos ópticos virtuais para a transmissão de sinal considerase sua atuação como labels na arquitetura GMPLS sobre redes híbridas WDM/OCDM. Assim, utilizase a denominação de labels para códigos OOC e comprimentos de onda.

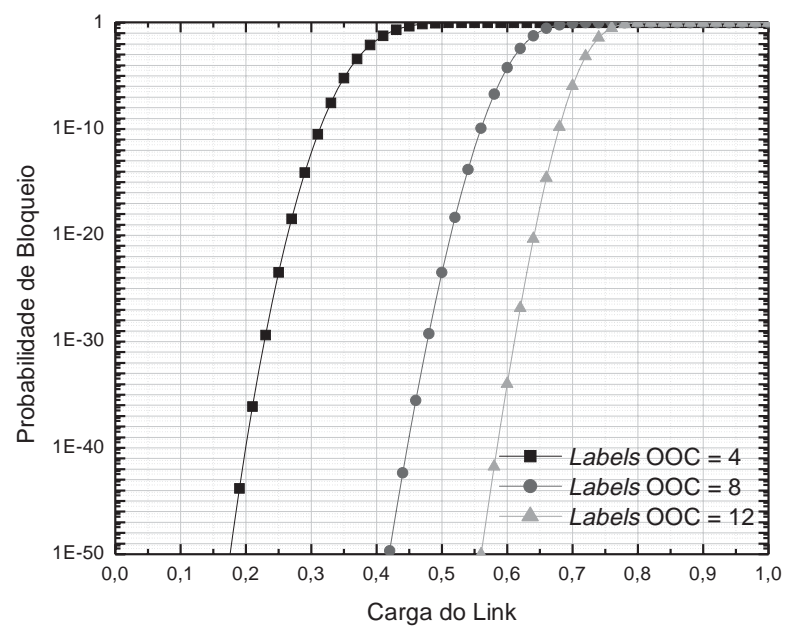

Figura 3. Probabilidade de bloqueio de estabelecimento de caminhos ópticos virtuais em função da carga do link.

Por meio da Fig. 3, observa-se que o aumento do número de códigos OOC (labels) aumenta a 
capacidade de utilização da rede. Por exemplo, para a probabilidade de bloqueio de $10^{-20} \mathrm{o}$ aumento de códigos OOC de 4 para 12 reflete num possível aumento da carga do link de 0,26 para 0,65. Assim, o aumento do número de códigos OOC representa uma alternativa para a expansão da capacidade da rede. Para melhor ilustrar esse conceito, é mostrada na Fig.4 a probabilidade de bloqueio de estabelecimento de caminhos ópticos virtuais em função do número de códigos OOC para uma rede com 8 enlaces e com uma carga de link de 0,3 .

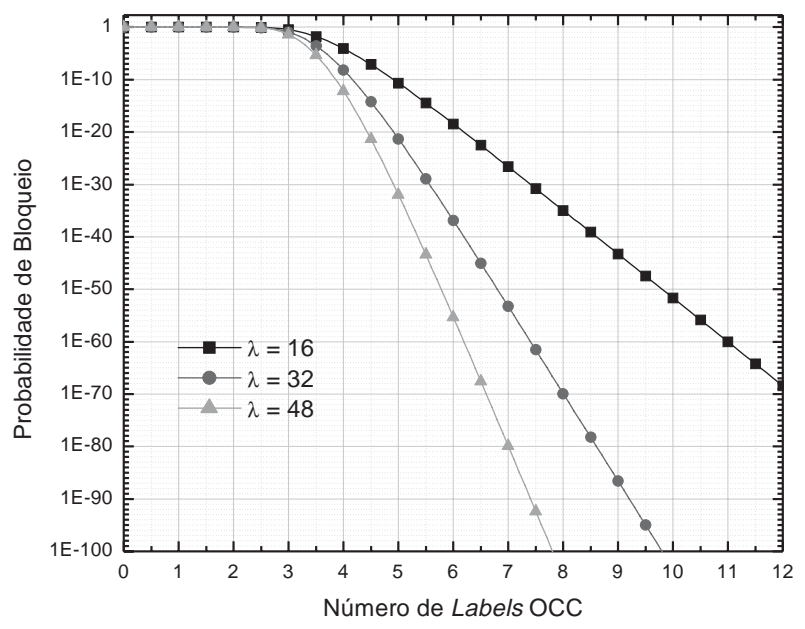

Figura 4. Probabilidade de bloqueio de estabelecimento de caminhos ópticos virtuais em função número de códigos OOC.

Observando a Fig.4, verifica-se que a probabilidade de bloqueio de estabelecimento de caminhos ópticos virtuais decresce rapidamente a partir da utilização de 3 códigos OOC. Este comportamento pode ser mais bem analisado realizando uma comparação com uma rede de 8 enlaces e carga de link igual a 0,3 , o qual possui capacidade de conversão de comprimento de onda em todos os nós. Se esta rede empregar 16 comprimentos de onda, obtém-se uma probabilidade de bloqueio de $10^{-6}$ (SUBRAMANIAM; AZIZOGLU; SOMANI, 1996) e (BARRY; HUMBLET, 1996). Essa mesma probabilidade de bloqueio poderia ser obtida utilizando 16 comprimentos de onda e 4 códigos OOC sem utilizar nenhum conversor de comprimento de onda na rede. Portanto, a configuração do número de comprimentos de onda e códigos OOC constitui um elemento de projeto que deve ser considerado neste tipo de rede. Dessa forma, na Fig. 5, ilustra-se o estudo da probabilidade de bloqueio de estabelecimento de caminhos ópticos virtuais em função número de comprimentos de onda e códigos OOC. Considerouse, para tanto,uma rede com 8 enlaces.

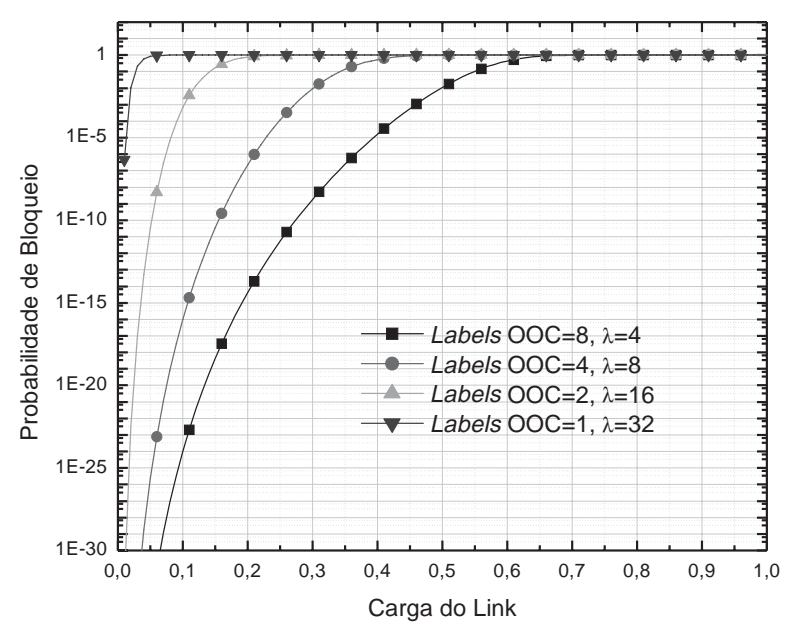

Figura 5. Probabilidade de bloqueio de estabelecimento de caminhos ópticos virtuais em função da carga do link.

Neste estudo foi mantido o número de labels igual a 32 por link (número de comprimentos de onda $\times$ número de códigos $\mathrm{OOC}=32$ ). Esta manutenção no número total de elementos de caminhos ópticos virtuais foi realizada para mostrar o desempenho da rede com um número limitado de recursos de roteamento. Por meio da Fig. 5, observa-se que os códigos OOC causam um maior impacto na diminuição da probabilidade de bloqueio de estabelecimento de caminhos ópticos virtuais que os comprimentos de onda. Este comportamento pode ser ilustrado observando que para uma carga de link de 0,3 , empregando 32 comprimentos de onda e 1 código OOC, a probabilidade de bloqueio de estabelecimento de caminhos ópticos virtuais é aproximadamente 1 . Por outro lado, se para a mesma carga de link forem empregados 4 comprimentos de onda e 8 códigos OOC, a probabilidade de bloqueio 
chegará a $10^{-7}$. Assim, o emprego de códigos OOC pode ser utilizado como estratégia de expansão da capacidade de redes ópticas WDM que estejam migrando para a arquitetura GMPLS utilizando a tecnologia OCDM. Outra forma de se aumentar o desempenho das redes WDM/OCDM é a distribuição de conversores de códigos ópticos OOC ao longo da rede (DURAND et al., 2005). Assim, é mostrada na Fig. 6, a probabilidade de bloqueio de estabelecimento de caminhos ópticos virtuais em função da carga do link para uma rede de 8 enlaces, 8 comprimentos de onda e 4 códigos ópticos por link. Foram analisadas as redes com fatores de conversão de códigos ópticos de $0,3,0,6$ e 1 . O fator de conversão é dado por:

$$
\text { Fator de Conversão }=\frac{K-1}{H-1}
$$

em que $K$ representa o número de nós com capacidade de conversão de códigos ópticos e $H$ é o número total de nós da rede. O fator de conversão representa a capacidade dos nós da rede de efetuarem a conversão de códigos ópticos e se considera que os conversores estejam uniformemente distribuídos pela rede (DURAND et al., 2005).

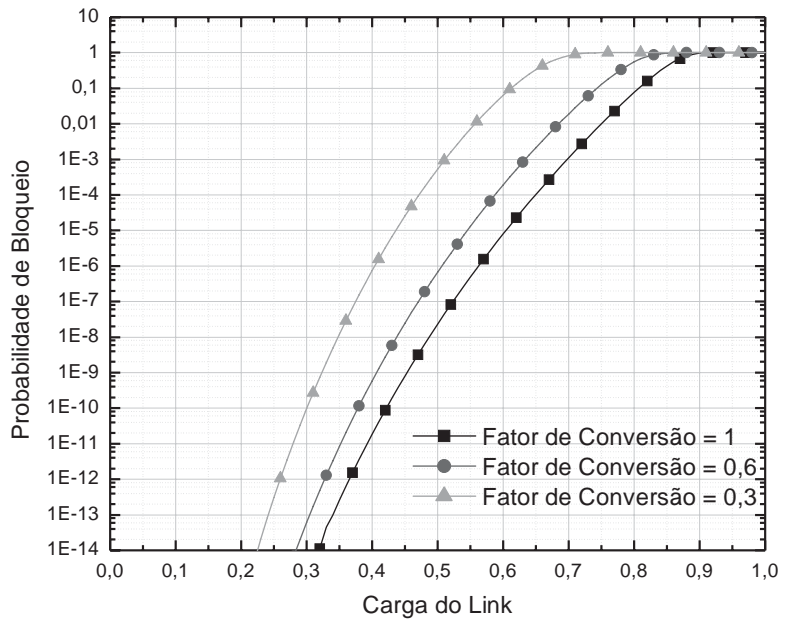

Figura 6. Probabilidade de bloqueio de estabelecimento de caminhos ópticos virtuais em função da carga do link.

O resultado obtido na Fig. 6 ilustra que o emprego de conversores de códigos OOC melhora o desempenho das redes ópticas, porém para melhor analisar o impacto dos conversores de códigos OOC ilustra-se na Fig. 7, a probabilidade de bloqueio de estabelecimento de caminhos ópticos virtuais em função do fator de conversão de códigos ópticos considerando uma rede com 8 enlaces e 8 comprimentos de onda.

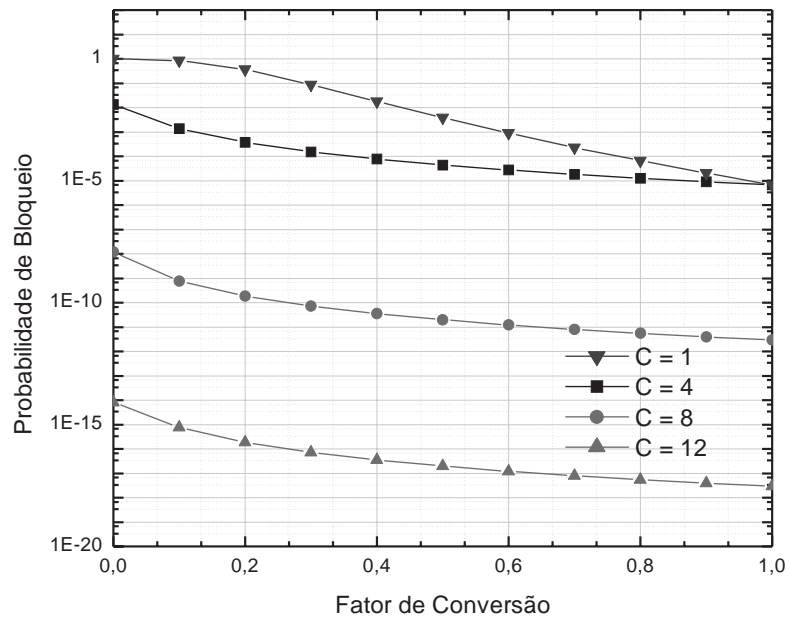

Figura 7. Probabilidade de bloqueio de estabelecimento de caminhos ópticos virtuais em função do fator de conversão de códigos ópticos para valores distintos de códigos OOC.

A Fig. 7 mostra que o aumento do fator de conversão diminui a probabilidade de bloqueio de estabelecimento de caminhos ópticos virtuais. No entanto, também se observa que o aumento de códigos OOC tem um grande impacto na diminuição da probabilidade de bloqueio de estabelecimento de caminhos ópticos virtuais. Isso pode ser ilustrado, por exemplo, considerando um fator de conversão igual a 0,6 . Nesse caso, a probabilidade de bloqueio de estabelecimento de caminhos ópticos virtuais diminui de $10^{-11}$ para $10^{-7}$, quando se aumenta o número de códigos OOC de 8 para 12. Por meio da Fig. 7, também é possível observar que mesmo que a rede possua capacidade de efetuar a conversão de códigos ópticos em todos os nós, o aumento do número de códigos OOC possui um impacto maior na diminuição da probabilidade de bloqueio. Por exemplo, a probabilidade de bloqueio de estabelecimento de caminhos ópticos virtuais para uma rede com 4 
códigos OOC e capacidade de conversão de comprimento de onda em todos os nós da rede é de $10^{-5}$. Por outro lado, uma rede com 8 códigos OOC sem capacidade de conversão de códigos ópticos em nenhum nó da rede apresentará uma probabilidade de bloqueio de estabelecimento de caminhos ópticos virtuais de $10^{-8}$. Porém, o aumento do número de códigos OOC apresenta um grande impacto na diminuição da probabilidade de bloqueio de estabelecimento de caminhos ópticos virtuais e, assim, verifica-se que o número de códigos OOC em cada enlace é limitado em função de efeitos de dispersão como PMD (Polarization Mode Dispersion), como é demonstrado em (DURAND et al., 2005). Portanto, para a definição do número de códigos OOC e do número de nós com capacidade de conversão de códigos OOC deve-se realizar um estudo da topologia da rede. Entretanto, observa-se que utilização de códigos OOC em conjunto com conversores de códigos ópticos pode representar uma alternativa para expansão da rede.

\section{Conclusões}

Neste trabalho, apresenta-se uma análise de redes ópticas híbridas WDM/OCDM (Wavelength Division Multiplexing/Optical Code-Division Multiplexing) que empregam comprimentos de onda e códigos ópticos OOC (Optical Orthogonal Code) para a comutação e roteamento no domínio óptico. Nesta análise, foram apresentadas as vantagens do emprego de labels compostos por códigos ópticos bem como a arquitetura de rede utilizada. Como resultados obtidos, observou-se que o emprego de códigos OOC juntamente com conversores de códigos ópticos tem um grande impacto na diminuição da probabilidade de bloqueio de estabelecimento de caminhos ópticos virtuais. Por outro lado, para estimar qual a configuração mais adequada com relação ao número de códigos OOC e número de nós com capacidade de conversão de códigos ópticos deve-se realizar uma análise da topologia da rede.
Nesta etapa de trabalho, consideraram-se caminhos ópticos virtuais formados por comprimentos de onda e códigos ópticos OOC, que equivale à rede comutada por meio de circuitos ópticos. As próximas investigações se concentrarão na análise de redes com arquitetura GMPLS (Generalized Multiprotocol Label Switching) que utilizam labels baseados em comprimento de onda e códigos ópticos para comutação na forma de pacotes e rajadas.

\section{Referências}

BANERJEE, A.; DRAKE, J.; LANG, J.; TURNER, B.; AWDUCHE, D.; BERGER, L.; KOMPELLA, K.; REKHTER, Y. Generalized Multiprotocol Label Switching: An Overview of Routing and Management Enhancements. IEEE Communications Magazine, New York, v. 39, n. 7, p. 144-150, jan. 2001.

BARRY, R.; HUMBLET, P. A. Model of Blocking Probability in All-Optical Network with and without Wavelength Charges. IEEE Journal on Selected Areas in Communication, New York, v. 14, n.5, p.858- 867, 1996.

BLUMENTHAL, D.; OLSSON, B. E; ROSSI, G.; DIMMICK, T.E; RAN, L.; MASANOVI, M.; LAVROVA, O.; DOSHI, R.; JERPHAGNON,O.; BOWERS, J.E; KAMAN, V.; COLDREN, L. A; BARTON, J.All-Optical Label Swapping Networks and Technologies. Journal of Lightwave Technology, New York, v.18, n.2, p.2058-2075, 2000.

CINCOTTI,G. Design of Optical Full Encoders/Decoders for Code-Based Photonic Routers. Journal of Lightwave Technology, New York, v.22, n.7, p.1642-1650, jul. 2004.

DJORDJEVIC, I.; VASIC,B. Combinatorial Constructions of Optical Orthogonal Codes for OCDMA Systems. IEEE Communications Letters, New York, v.8, n.6, p. 391-393, jun. 2004.

DURAND, F. et al. Análise de Redes Ópticas WDM/ OCDM. In: SIMPÓSIO BRASILEIRO DE TELECOMUNICAÇÕES-SBT'04, 21., 2004, Belém. Anais... BELÉM: Sociedade Brasileira de Telecomunicações, 2004.

DURAND, F. et al. Analysis of Hybrid WDM/OCDM Networks with All-Optical Code Converters. In: INTERNATIONAL MICROWAVE AND OPTOELETRONICS CONFERENCE, 2005, Brasília. Proceedings... Brasília: Cria Design, 2005.

KITAYAMA, K.; WADA, N.; SOTOBAYASHI, H. Architectural Considerations for Photonic IP Router Based upon Optical Code Relation, Journal of Lightwave Technology, New York, v.18, n.12, p.1834 - 1844, 2000. 
KITAYAMA, K.; WADA, N. Versatile Optical Code-Based MPLS for Circuit, Burst, and Packet Switchings. Journal Of Lightwave Technology, New York, v.21, n.11, p.27532764, 2003.

LIU, K.; LIU, C. D.; PASTOR, J. L; ROY, A. WEI, J. Y. Performance and Testbed Study of Topology Reconfiguration in IP over Optical Networks. IEEE Transactions on Communications, New York, v.50, n.10, p. 1662-1679, 2002.

MAJUMDER, S. P.; AZHARI, A.; ABBOU, F. M. Impact of Fiber Chromatic Dispersion on the BER Performance of an Optical CDMA IM/DD Transmission System. IEEE Photonics Technology Letters, New York, v.17, n.6, p.1340 - 1342, 2005.
MURTHY, C. S.; GURUSAMY, M. WDM Optical Networks: Concepts, Design, and Algorithms. New Jersey: Upper Saddle River, 2002.

SUBRAMANIAM, S.; AZIZOGLU, M.; SOMANI, A. K. All-Optical Networks with Sparse Wavelength Conversion. IEEE/ACM Transactions on Networking, New York, v.4, n.4, p.544-557, 1996.

WEN, Y. G.; ZHANG, Y.; CHEN, L. K. On Architeture and Limitation of Optical Multiprotocol Label Switching (MPLS) Networks Using Optical-Orthogonal-Code (OOC)/ Wavelength Label. Optical Fiber Technology, v.8, n.1, p. 43-70, 2002.

ZHANG, Y; JI, Yuefeng; CHEN, Xue. Code Routing Technique in Optical Network. Beijing: Beijing University of Posts \& Telecommunications, 1999. 